\title{
Safety Guidelines for the Laser Removal of Dental Calculus
}

\author{
Japanese Society for Laser Dentistry
}

\section{Foreword}

These guidelines will outline the background, principles, clinical and basic evidence of the use of lasers in dentistry, especially on calculus removal (scaling). This article will also discuss the actual clinical laser application and safety measures ${ }^{1)}$.

\section{Background: the significance of calculus removal in periodontal treatment and the role of laser application.}

The fundamental concept of periodontal treatment is to restore the biocompatibility of root surfaces affected with periodontal disease and encourage periodontal tissue to re-adhere to the root surface. Root surfaces affected with periodontal disease have plaque and calculus adhering to them, and may also be contaminated by harmful substances such as bacterial endotoxins and metabolites. In order for periodontal tissue to heal successfully, complete removal of these substances is required. Areas of plaque attached to the root surface within the root pocket form a bacterial bio-film which blocks any systemic effect of oral or other antibiotics, and mechanical destruction is the only method for its removal ${ }^{2)}$.

The conventional method of removing deleterious substances from the root surface is mechanical scaling root planing (SRP) with manual scalers ${ }^{3}$ ). Some practitioners use ultrasonic scalers or air-scalers ${ }^{4}$ ) along with conventional manual scalers which alleviate some of the burden of the procedure, however the SRP procedure still remains one that is time consuming and requires skill and experience. It has been reported that conventional SRP cannot completely remove the bacterial deposits adhering to the root surface and also that

Addressee for Correspondence:

Japanese Society for Laser Dentistry

Secretariat

Kouku hoken Kyoukai 1-43-9 Komagome,

Toshima, Tokyo, Japan

Tel: +81-3-3947-8891 / Fax: +81-3-3947-5341

E-mail: gakkai18@kokuhoken.or.jp concavities, furcations and grooves of the root surface and distal planes of molars are difficult to access, and that there are limitations to conventional mechanical procedures. Elimination of microbes within the periodontal pocket can be achieved with local or systemic administration of antibiotics, but there always remains the problem of drug allergies, and increased drug resistance of the bacteria due to prolonged use of drugs. Therefore, presently, lasers are being clinically applied as either a complementary method or as an alternative method to conventional scaling. Lasers are known for their bactericidal and tissue ablative effects and are being used not only for calculus removal, but also for debridement of the root surfaces ${ }^{5-7}$ ).

\section{Er:YAG laser and the application of lasers to periodontally diseased roots}

Originally the Nd:YAG laser was applied for the debridement of periodontally diseased root surfaces. In the case of the Nd:YAG laser the wavelength of 1064 $\mathrm{nm}$ is highly absorbed by dark chromophores. The Nd:YAG laser was reported to be capable of removing dark colored sub-gingival calculus and has been used clinically 8-10). However, lasers such as the Nd:YAG laser and also the $\mathrm{CO} 2$ laser, are basically incapable of ablating biological hard tissues because these targets lack the specific chromophores, namely dark-colored materials for the Nd:YAG and water for the CO2. High output laser irradiation for calculus removal or of the root surface is known to cause thermal damage such as carbonization and melting of enamel and dentin, and caution is advised 11,12). On the other hand, a report by Hibst and Keller ${ }^{13)}$ and a report by Kayano ${ }^{14)}$ in 1989 have provided basis that ablation of hard tissue is possible using the Er:YAG laser and made possible the laser treatment of caries ${ }^{15-19)}$. In the field of periodontology, laser ablation of hard tissue, such as calculus removal and bone tissue management, became possible by the mid-' 90 s $5,7,19,20$ ). 


\section{The characteristics of the Er:YAG laser and the mechanism behind vaporization of calculus}

The Er:YAG laser is a solid state laser first developed by Zharikov, et al. ${ }^{21)}$ in 1974. It is a pulsed laser with a wavelength of $2.94 \mu \mathrm{m}$. Among the lasers used presently in dentistry, indeed in any aspect of surgery and medicine, the Er:YAG is the most highly absorbed by water, showing theoretical absorption 10 times more than that of CO2 laser, and 15,000 to 20,000 times more than that of the Nd:YAG laser 22,23 ). Therefore the affinity of the Er:YAG laser light to living tissue is extremely high and ablation of hard tissue as well as soft tissue is possible. The high absorption by water limits collateral thermal damage to the surrounding tissue. In the case of hard tissue, the amount of water contained within the tissue is small and heat generation is present but can be controlled with water irrigation 24). Many systems used the irrigation water as the actual target, and eliminate the hard tissue through kinetic energy delivered by the microburst principle whereby the explosive force of vaporization of the thin film of water is transferred to the hardt tissue, thereby ablating it. The thickness of the denatured layer of the root cementum and dentin following Er:YAG laser irradiation of the root surface under water irrigation is reported to be 5-15 $\mathrm{m}^{25-27)}$. The mechanism behind ablation is firstly through photothermal evaporation where the light energy is absorbed by water in the hard tissue itself and in other organic substances and secondly by the mechanical effect already mentioned, bringing about tissue ablation through the microburst principle, also known as the microexplosion concept where the water vapor pressure build-up created by the extremely violent evaporation of water exceeds the threshold of the tissue. Ablation by microexplosion is referred to as photomechanical ablation or thermomechanical ablation 28, 29). Calculus is a multi-porous calcified substance, which contains water not only as a constituent of the substance but also within its pores. Hence, in normal biological conditions, calculus is one of the more easily ablated tissues using the Er:YAG. A more recent laser based on an Er,Cr:YSGG medium emits laser light similar to the Er:YAG laser at a wavelength of $2.78 \mu \mathrm{m}$, and has been reported to have similar efficacy concerning calculus removal ${ }^{30}$ ).

\section{Basic evidence behind calculus removal with the Er:YAG laser}

1) Efficacy of calculus removal and influence of laser energy on the sub-calculus root surface

Using the Er:YAG laser, calculus is readily ablated at a lower energy level (11-19 J/ $\mathrm{cm}^{2} /$ pulse) compared to that of ablating tooth hard tissue 5,25,31-34). Irradiation under water irrigation results in a popping sound as the calculus is being ablated. The laser irradiated surface of the calculus shows no sign of thermal damage such as carbonization or melting, and it is proposed that the calculus is mechanically disintegrated ${ }^{25)}$. An in vitro study on the efficiency of laser calculus removal using extracted teeth as the target showed that the rate of calculus removal at a contact tip output of $40 \mathrm{~mJ} /$ pulse (energy density of $14.1 \mathrm{~J} / \mathrm{cm}^{2} /$ pulse) and a pulse rate of $10 \mathrm{pps}(\mathrm{Hz})$ is equivalent to that of an ultrasonic scale. When the pulse rate is changed to 20-30pps, the rate of the removal with the laser is increased and the efficiency of the laser becomes greater than that of the ultrasonic scaler.

It is basically impossible to use the Er:YAG laser for the complete and selective removal of all calculus. Thus, calculus adherent to the enamel is contraindicated as a target due to the thermal damage and ablation of the sub-calculus enamel ${ }^{35)}$. In the case of subgingival calculus of the root surface, not only removal of the calculus but also a removal of the contaminated cementum by root plaining is also required. Therefore, ablation of the superficial layer of the cementum is permissible, and laser treatment is indicated for subgingival calculus. However, care must be taken during the laser irradiation, to not overly ablate the cementum by making sure that the contact tip of the laser moves parallel to the root surface or irradiating the laser at certain constant angles to the target $5,25,36$ ). Preservation of the cementum is possible $25,31,36$ ) by controlling the laser irradiation method and output. While nonsurgical treatment such as SRP completely removes the cementum, there are reports stating that Er:YAG laser ablation preserves cementum ${ }^{37}$ ), or that cementum loss is comparable to conventional methods ${ }^{32}$ ). There is also a report stating that the loss of cementum is greater in laser ablation cases 33). Such differences in results are considered to be caused by the differing conditions of laser irradiation such as the conditions and the compatibility of the contact tip of the laser to root surface, output power, irradiation maneuvers and method. 


\section{2) Heat generation during laser irradiation and its effect on the root surface and dental pulp}

The effect of heat generation on the root surface and pulp is minimal when laser energy is delivered under conditions of water irtrigation 25,31 ) and no thermal damage such as carbonization of the root surface is seen. However, when a thin layer of cementum is ablated, the ablated surface undergoes a micro-structural change which appears white when dried $27,31,35,38$ ). The outmost layer of the micro-structural change following laser ablation shows a marked decrease in organic substances ${ }^{39)}$, while the deeper layers show changes limited to a few micrometers in depth that is believed to be caused by thermal denaturation 25,27). However Er:YAG laser irradiation under water irrigation shows almost no sign of formation of toxic substances due to carbonization ${ }^{39}, 40$ ) as seen with the $\mathrm{CO} 2$ laser. Er:YAG laser irradiation delivers bactericidal effects at extremely low energy levels ${ }^{41)}$ and also removes and detoxifies the endotoxins on the irradiated root surface 42,43 ). There are no smear layers on the laser irradiated surface 25,44$)$ and such advantageous effects as the bactericidal, detoxifying effect ${ }^{44)}$ of the laser are something that cannot be expected by conventional mechanical methods, and are furthermore considered to enhance the wound healing of the periodontal pocket.

Concerning the effect of lasers on the dental pulp, it has been reported that the damage when the laser is used for cavity preparation, a procedure that uses the laser at a much higher output, is comparable to that of conventional high speed burring for the same procedure 45,46$)$. It is therefore assumed that damage to the pulp when lasers are used for calculus removal is minimal if any at all, always considering the direction and method of laser irradiation. In actual animal studies, no histological change of the pulp was observed when laser was used for the debridement of the root surface during flap surgery ${ }^{47)}$.

\section{3) The effect on the adhesion of cells and} tissue to the laser irradiated root surface Concerning the adhesion of periodontal tissue to the Er:YAG ablated surface, there have been numerous in vitro studies conducted comparing that of the Er:YAG debrided surface and conventional SRP with a curette. Multiple studies report the significantly higher cellular adhesion associated with the laser irradiated surfaces ${ }^{48-50)}$. On the other hand, studies conducted using healthy cementum showed that adhesion decreased in the case of cementum manifesting micro-structural changes due to laser irradiation, however this decrease was reversible by treatment of the root surfaces with tetracycline hydrochlorates or ethylenediaminetetraacetic acid (EDTA) ${ }^{51)}$.

An in vitro animal study conducted by Schwarz, where wound healing following nonsurgical treatment was assessed, showed no severe thermal damage to the root surface after debridement with the Er:YAG laser and that the ablation was contained within the cementum with new cementum formation similar or greater than that following SRP 52). An animal study of surgical treatments conducted by Mizutani, where the Er:YAG laser was used for the debridement of both the root surface and boney defect, showed that laser irradiation to the root surface had no hindering influence on tissue adhesion. Although there were some remnants of heat denaturation, in general most of the denaturation layer had been resorbed and new cementum formation along with connective tissue attachment comparable to that of SRP was observed ${ }^{47)}$. Excellent results of clinical studies that will be mentioned later, and long term stability of the results, have shown that laser irradiation to the root surfaces does not impede tissue adhesion, at least in a clinical context. However the evidence concerning laser ablated root surfaces and periodontal tissue adhesion is still insufficient and future in vivo studies are warranted.

\section{Clinical evidence for Er:YAG application for the debridement of the root}

\section{1) The application of lasers in non-surgical} treatment of the periodontal pocket

Schwarz, et al. ${ }^{53)}$ conducted a randomized controlled trial (RCT) of a split-mouth design, comparing non-surgical pocket treatment with the Er:YAG laser and that with conventional SRP using a curette. The results showed that the procedural time for the laser group was shorter than the SRP group and that pocket reduction at 6 months of post-treatment was similar for both groups. The improvements of both attachment level and bleeding on probing (BOP) were significantly higher in the laser group compared to the SRP group and these results were maintained for 2 years ${ }^{54)}$. In another report by the same authors, root planning with a curette in addition to laser treatment had no beneficial impact on the results ${ }^{55)}$. Sculean, et al. 
reported that when the Er:YAG laser was compared to ultrasonic scalers, treatment results at 6 months were comparable ${ }^{56)}$, while Crespi, et al. reported that at 1 and 2 years post treatment, the laser treated group showed significantly greater improvement compared to ultrasonic scaler treated group 57 ). Tomasi, et al. reported the application of the Er:YAG laser for periodontal maintenance which resulted in significantly greater improvement of the laser treated group at 1-months post-treatment, but at 4-months post-treatment, both the improvement levels and the bacterial levels were similar for both laser and non-laser groups. They also reported that treatment discomfort was less in the laser treated group ${ }^{58)}$. However, others have reported that ultrasonic scaling results in greater decrease of bacteria compared to Er:YAG laser treatment, and that patients preferred ultrasonic scaling over laser treatment ${ }^{59)}$. There are others reporting that the removal rate of calculus using lasers blindly in the pocket becomes less than that using the curette ${ }^{37)}$, or even others concluding that lasers should be used as an adjunct to conventional mechanical methods ${ }^{60}$ ). Thus, the evaluations still vary.

Although there are some reports, as listed above, concluding that exclusive treatment with the Er:YAG laser shows significantly greater improvements in the long term, if not at least similar results compared to SRP, a consensus has not yet been reached ${ }^{6}$.

\section{2) Application in periodontal surgical treat- $\underline{\text { ment }}$}

In application of the Er:YAG laser in periodontal surgery, the laser is used in an open visual field. Confirmation of calculus deposition and adaptation of the contact tip of the laser to the calculus is easily done the laser can be aimed and the target tissue irradiated with much more control, resulting in less ablation of the root surface.

Thus, debridement of the root surface with the Er:YAG laser in an open visual field is extremely easy and highly reliable. Furthermore, due to its high performance in ablation of soft tissue and the applicability in boney tissue, the main purpose of applying the Er:YAG laser is to ablate the diseased granulation tissue in the bone defects during flap surgery ${ }^{7,47)}$. An animal study by Mizutani, et al. demonstrated the safe and effective debridement of the root surface and granulation tissue in a flap surgery. This study also suggested the possibility of enhancement of osteogenesis by laser irradiation
47)

In a clinical trial conducted by Sculean et al., a comparison of Er:YAG laser irradiation during flap surgery for the debridement of the root surface and bone defect with that with curettes and ultrasonic devices was reported. Although no significant clinical differences were seen between the laser and non-laser groups, the authors concluded that Er:YAG laser was a suitable alternative for periodontal surgery ${ }^{61)}$. Gaspric, et al. applied the Er:YAG laser for the debridement of the root surface and bone defects and concluded that clinical improvement was significantly greater in the laser treated group until up to 3-years post treatment, compared to the group receiving flap surgery with conventional mechanical methods. In this study, a significantly greater decrease of pocket depth and a significant increase in attachment gain were detected in the laser group ${ }^{62)}$. In the application of the Er:YAG laser in periodontal surgery, debridement of the root surface is easily performed in an open visual field and the wound healing is equal to or better than conventional mechanical treatment methods. In order to accumulate further evidence, studies and trials in the future should include multicenter studies with large number of subjects, which are designed for the heterogeneous analysis of the treatment effect.

\section{Laser scaling (calculus removal) proce- dure with the Er:YAG laser}

\section{1) The requirements for the dentists and dental facility performing the laser removal of calculus}

Dentists performing the procedure must have thorough knowledge of and training in periodontal treatment and laser treatment of oral tissue. They are also required to have knowledge of, and have been trained in and are capable of operating the laser hardware and devices. Therefore the dentists should be certified as a specialist or an instructor by the Japanese Society for Laser Dentistry, or a certified dentist by the NPO Japan Society for Laser Surgery and Medicine, certified specialist or instructor by the NPO Japanese Society for Periodontology. A suitable dental facility would be one where a specialist listed above works full time and possesses adequate hardware and machinery and has taken necessary safety measures such as light shielding and protection. Therefore an independent laser treatment room or a partitioned laser treatment unit must be provided and all laser treatment must be 
performed within these controlled areas.

\section{2) Laser devices that are approved for the application of scaling}

Presently the only laser device approved for scaling by the Ministry of Health, Labour and Welfare in Japan is the Er:YAG laser. There are 4 commercially available Er:YAG laser devices for periodontal usage: Erwin ${ }^{\circledR}$, Erwin AdvErL ${ }^{\circledR}$, Dentlite ${ }^{\circledR}$, and DentLite $30^{\circledR}$

\section{3) Calculus deposition subject to laser scal- ing}

The Er:YAG laser is capable of ablating tooth substance, therefore removal of calculus formed over the enamel is contraindicated. Calculus of the subgingival root surface, or exposed root surface due to gingival recession, or root surface exposed during periodontal surgery are indicated.

\section{4) Safety measures and precautions for}

\section{clinical application}

(1) Pre-operative checks

The laser hardware must be both visually and functionally inspected prior to the procedure. Attach the contact tip and handpiece sterilized with an autoclave to the device and perform a functional check such laser output and irrigation water volume. If at the time of the power check, the laser output is unusually low, all connections from the laser mainframe to the handpiece cover and to the contact tip need to be examined, the collective lens should be checked for any contamination and the output power should be checked again.

The contact tip of the laser is disposable and the tip should be examined and changed periodically. Spare contact tips must be prepared at all times since the probe may be damaged during the procedure which might cause a decrease in laser output. Be sure to test fire the laser into the dental vacuum and test laser emission and condition of the air spray, before actually using the laser in the mouth.

(2) Intraoperative precautions.

a. Protection of the eyes with protective goggles.

Lasers are effective new devices for dental treatment but differ greatly from conventional mechanical devices in that laser energy may cause damage from a distance, with the device never coming in contact with tissue.
Such is the case in misfiring of the lasers and special attention should always be paid to correct aiming and firing. Therefore when a laser is being used, the patient, the dentist and assistant are all required to wear protective goggles with a suitable optical density (OD) appropriate for the wavelength of the laser energy being used. Attention must also be paid to reflected light from dental mirrors and metal restorative material 5, 63, 64).

b. Setting of the optimum laser parameters When using a $600 \mu \mathrm{m}$ diameter contact tip, the panel output should be set to $40-80 \mathrm{~mJ} /$ pulse (resulting tip output $20-40 \mathrm{~mJ} /$ pulse) as a rough standard (energy density of 7.1-14.2 $\mathrm{J} / \mathrm{cm}^{2} /$ pulse ), and a repetition rate $10-30 \mathrm{pps}$ $(\mathrm{Hz})$. Select the appropriate contact tip according to the condition of the periodontal pocket and root surface. In order to control heat generation during hard tissue ablation, water irrigation with the air spray is necessary. Set air and water volume to an appropriate setting. However when using the air spray within the periodontal pocket, the setting of the air spray should be lowered to a minimum or even turned off since there is a possibility of the air spray causing subcutaneous emphysema. In the case of periodontal surgery there is no possibility of emphysema, but the irrigation water must be changed to sterile normal saline.

c. Detection and identification of the calculus According to the usual procedure, subgingival calculus within the periodontal pocket is detected using a periodontal pocket probe. After the procedure, the probe is inserted into the pocket again to confirm the removal of the calculus and the irregularity of the root surface. Identification and confirmation during periodontal surgery is simple since the root surface is exposed to direct vision. Local anesthetic is necessary for surgery but in the case of non-surgical procedures it is up to the severity of the cases and/or patient' pain perception and threshold.

d. Selection of appropriate laser irradiation method

Calculus can be removed by either contact or non-contact irradiation. When using the contact method the contact tip does not need to be firmly pressed against the calculus, but should rather be held in light contact. When 
removing calculus from the root surface, use the contact tip always holding it parallel or at a constant slight angle up to $30^{\circ}$ to the root surface, always moving it up and down or right to left in order to avoid over-irradiating any single point. Inappropriate laser irradiation may cause unwanted ablation and damage and care must be taken to avoid this.

e. Effects on the surrounding tissue

Since Er:YAG laser is associated with very superficial absorption characteristics because of the affinity of its wavelength with water, 19) basically the penetration depth of the laser energy is small and the risk of unexpected deep tissue damage is very low, making procedures carried out with this laser clinically very safe. However care should still be taken to avoid any thermal effect on the periosteum.

Ablation of the periodontal tissue attached to the area of the pocket such as the bottom or lateral margins of the pocket should be avoided. As for the effect on the dental pulp, if the output, irradiation method, and water irrigation are correctly employed, thermal damage to the pulp can be basically avoided.

f. Prevention of damage due to misfiring Attention must be paid to all tissue within the range and direction of the laser energy. Protection of non-target tissue by shielding and other measures should be provided if necessary. Misfiring of lasers due to careless stepping on to the footswitch must be avoided. Each laser emission should be intentional and fired with care.

g. Effective evacuation of ablation-related fumes The explosive nature of ablation of intrapocket tissue with the Er:YAG laser energy causes formation of a plume of particulate matter, including blood and ablated tissue, to scatter from the pocket. The use of an effective smoke evacuator is necessary along with personnel protection with face guards.

h. In the case of an adverse event If and when an adverse event occurs to either the patient or connected with the laser device, abort the procedure immediately by either releasing the foot pedal or by pressing the emergency stop button and attend to the patient or find the cause of the abnormality. If the laser tip is broken within the periodontal pocket, make sure that all pieces are recov- ered and removed from the pocket.

(3) Post-operative precautions

a. Examination of the treated area

Check for abnormal bleeding and pain. Observe the degree of damage to the tissue and whether or not there is excessive damage to the tissue and finally check for complete hemostasis.

b. Additional treatment of the irradiated root surface

Presently, it is thought that additional mechanical or chemical treatments of laser ablated root surfaces are unnecessary. However, chemical treatment performed under direct vision following periodontal surgery is thought to enhance post-operative wound healing.

c. Sterilization of the used equipment

After the procedure, remove the handpiece cover and contact tip from the device, wash thoroughly and submerge in protein dissolving agent. After each part is dried, it is packaged separately and sterilization is performed with an autoclave. At this stage, check for damage to the contact tip before it is packaged.

d. Laser safety officer and periodic inspection The facility is required to appoint a Laser Safety Officer (LSO), to ensure that the LSO fulfills all of his or her obligations, and have the laser device periodically inspected.

\section{Afterword}

Conventional mechanical treatment of periodontally diseased root surfaces within the periodontal pocket has limitations such as accessibility and bactericidal effect. The treatment outcome is strongly influenced by technique, and the procedure is time consuming. High hopes arose for laser application to be a new treatment method when it first appeared. More and more evidence concerning the safety and efficacy of calculus removal with the Er:YAG laser is accumulating while the number clinical laser applications in surgical and non-surgical treatment is slowly growing ${ }^{7)}$. However there are still several problems or questions that need to be solved such as the long term effect and the level of calculus removal achieved by exclusively lasereffected non-surgical treatment or the effect of combination treatment with mechanical methods. Presently there are too few comparative studies between laser and conventional treatments and the true clinical efficacy has not been made clear. Furthermore clinical 
studies based on RCT's, systematic review of the studies and meta-analyses are necessary. The clinical use of the laser should be performed by those who have already acquired the skill and knowledge of conventional mechanical methods. Laser should be applied

\section{References}

1: Aoki A, Mizutani K, Watanabe H, et al.: Position paper: Dental calculus removal with lasers (edit. by NPO Japanese Society of Periodontology, Japanese Society for Laser Dentisitry). J Jpn Soc Perio. 52(10):180-190, 2010. J Jpn Soc Laser Dent. 21(2): 100-109, 2010 (in Japanese).

2: Darveau RP, Tanner A, Page RC: The microbial challenge in periodontitis. Periodontol, 2000. 14: 12-32, 1997.

3: Cobb C: Clinical significance of non-surgical periodontal therapy: an evidence-based perspective of scaling and root planning. J Clin Periodontol, 29 (Suppl 2): 6-16, 2002.

4: Oda S, Nitta H, Setoguchi T, Izumi Y, Ishikawa I: Current concepts and advances in manual and power-driven instrumentation. Periodontol, 2000. 36: 45-58, 2004.

5: Aoki A, Sasaki K, Watanabe H, Ishikawa I: Lasers in non-surgical periodontal therapy. Periodontol, 2000, 36: 59-97, 2004.

6: Cobb C: Lasers in periodontics: a review of literature. J Periodontol. 77(4): 545-564, 2006.

7: Ishikawa I, Aoki A, Takasaki AA, et al.: Application of lasers in periodontics: true innovation or myth? Periodontol, 2000. 50: 90-126, 2009.

8: Ishikawa K, Fukuda M, Minoura S, et al.: A basic study on sub-gingivial calculus removal with the Nd:YAG laser. Japanese Journal of Conservative Dentistry. 36: 902-909, 1993.

9: Arcoria C, Vitasek-Ascoria B: The effects of lowlevel energy density Nd:YAG irradiation on calculus removal. J Clin Laser Med Surg. 10(5): 343-347, 1992.

10: Noguchi T, Sanaoka A, Fukuda M, et al.: Combined effects of Nd:YAG laser irradiation with local antibiotic application into periodontal pockets. J Int Acad Periodontol. 7(1): 8-15, 2005.

11: Tucker D, Cobb C, Rapley J, et al.: Morphological changes following in vitro $\mathrm{CO} 2$ laser treatment of calculus-ladened root surfaces. Laser Surg Med. 18(2): 150-156, 1996.

12: Morlock B, Pippin D, Cobb C, et al.: The effect of Nd:YAG laser exposure on root surfaces when used as an adjunct to root planing: an in vitro with appropriate skills and prudence, only with a complete understanding on the part of the clinician of the effect of lasers on soft and hard tissue and with evidence based on the results of scientific studies.

study. J Periodontol. 63(7): 637-641, 1992.

13: Hibst R, Keller U: Experimental studies of the application of the Er: YAG laser on dental hard substances: I. Measurement of the ablation rate. Lasers Surg Med. 9(4): 338-344, 1989.

14: Kayano T, Ochiai S, Kiyono K, et al.: Effects of Er:YAG laser irradiation on human extracted teeth. Kokubyo Gakkai Zasshi. 56: 381-392, 1989 (in Japanese, English abstract).

15: Kumazaki M, Toyoda K: Removal of hard dental tissue (cavity preparation) with the Er: YAG laser. J of Jpn Soc Laser Dent. 6(1): 16-24, 1995 (in Japanese, English abstract).

16: Takizawa M, Aoki S, Takase Y, et al.: Clinical evaluation of the Er:YAG laser for cavity preparation",Jpn J Conserv Dent 38: 1035-1047, 1995 (in Japanese, English abstract).

17: Matsumoto M, Nakamura Y, Mazeki K, Kimura Y: Clinical dental application of Er:YAG lasers for class V cavity preparation. J Clin Laser Med Surg. 14(3): 123-127, 1996.

18: Keller U, Hibst R: Effects of Er:YAG laser in caries treatment: A clinical pilot study. Lasers Surg Med. 20(1):32-38, 1997.

19: Katoh J, Awatsu K, Shinoki T, Moriya K: Laser dentistry from the fundamentals. Ishiyaku- Shuppan, Tokyo, 2003.

20: Aoki A, Andoh Y, Watanabe H, Ishikawa R: Application of lasers for the treatment of diseased root surfaces in periodontics. J Japanese Society for Laser Dentistry. 12(2): 109-117, 2001.

21: Zharikov E, Zhecov V, Kulevskii L, et al.: Stimulated emission from Er3+ ions in yttrium aluminum garnet crystals at $=2.94 \mathrm{~m}$. Sov J Quantum Electron. 4(8): 1039-1040, 1975.

22: Hale G, Querry M: Optical constants of water in the 200-nm to 200-micron wavelength region. Appl Opt. 12(3)]: 555-563, 1973

23: Niemz M: Laser-tissue interaction. Fundamentals and applications. Berlin, Springer-Verlag, 1996.

24: Burkes E, Jr., Hoke J, Gomes E, Wolbarsht M: Wet versus dry enamel abrasion by Er:YAG laser. J Prosthet Dent. 67(6): 847-851, 1992.

25: Aoki A, Miura M, Akiyama F, et al.: In vitro evalua- 
tion of Er:YAG laser scaling subgingival calculus in comparison with ultrasonic scaling. J Periodont Res. 35(5): 266-277, 2000.

26: Aoki A, Ishikawa I, Yamada T, et al.: Comparison between Er:YAG laser and conventional technique for root caries treatment in vitro. J Dent Res. 77(6): 1404-1414, 1998.

27: Fujii T, Baehni P, Kawai O, et al.: Scanning electron microscopic study of the effects of Er:YAG laser on root cementum. J Periodontol. 69(11): 1283-1290, 1998.

28: Koort H, Frentzen M: Laser effects on dental hard tissue. In: Miserendino 1, Pick R, eds. Lasers in Dentistry, vol Chicago, Quintessence Publishing Co, Inc..57-70, 1995.

29: Seka W, Featherstone J, Fried D, et al.: Laser ablation of dental hard tissue: from explosive ablation to plasma-mediated ablation. Proc SPIE. 2672: 144158, 1996.

30: Ting C, Fukuda M, Watanabe T, et al.: Effects of Er,Cr: YSGG laser irradiation on the root surface: morphologic analysis and efficiency of calculus removal. J Periodonotol. 78(11): 2156-2164, 2007.

31: Aoki A, Ando Y, Watanabe H, Ishikawa I: In vitro studies on laser scaling of subgingival calculus with an erbium:YAG laser. J Periodontol. 65(12): 1097-1106, 1994.

32: Folwaczny M Mehl A, Haffner C, et al.: Root substance removal with Er:YAG laser radiation at different parameters using a new delivery system. J Periodontol. 71(2):147-155, 2000.

33: Frentzen M, Braun A, Aniol D: Er:YAG laser scaling of diseased root surfaces. J Periodontol. 73(5):524530, 2002.

34: Schwartz F, Sculean A, Berakdar M, et al.: In vivo and in vitro effects of an Er:YAG laser, a Ga AlAs diode laser, and scaling and root planning on periodontally diseased root surfaces: a comparative histologic study. Lasers Surg Med. 32(5): 359-366, 2003.

35: Aoki A, Ishikawa I: The application of Er:YAG laser for the removal of calculus. Shika-Journal. 39(3): 279-287, 1994.

36: Folwaczny M, Thiele L, Mehl A, Hickel R: The effect of working tip angulation on root substance removal using Er:YAG laser radiation: an in vitro study. J Clin Periodontol. 28(3):220-226, 2001.

37: Eberhard J, Ehlers H, Falk W, et al. : Efficacy of subgingival calculus removal with Er:YAG laser compared to mechanical debridement: an in situ study. J Clin Periodontol .30(6):511-518, 2003.

38: Israel M, Cobb C, Rossman J, Spencer P: The effects of CO2, Nd:YAG and Er:YAG lasers with and without surface coolant on tooth root surfaces. An in vitro study. J Clin Periodontol. 24(9):595-602, 1997.

39: Sasaki K, Aoki A, Masuno H, et al.: Compositional analysis of root cementum and dentin after Er:YAG laser irradiation compared with $\mathrm{CO} 2$ lased and intact roots using Fourier transformed infrared spectroscopy. J Periodont Res. 37(1): 50-59, 2002.

40: Spencer P, Cobb C, McCollum M, Wieliczka D: The effects of $\mathrm{CO} 2$ laser and Nd:YAG laser with and without water/air surface cooling on tooth root structure: correlation between FTIR spectroscopy and histology. J Periodont Res. 31(7): 453-462, 1996.

41: Ando Y, Aoki A, Watanabe H, Ishikawa I: Bactericidal effect of erbium YAG laser on periodontopathic bacteria. Lasers Surg Med. 19(2): 190200, 1996.

42: Yamaguchi H, Kobasyashi K, Osada R, et al.: Effects of irradiation of an erbium: YAG laser on root surfaces. J Periodontol. 68(12): 1151-1155, 1997.

43: Sugi D, Fukuda M, Minoura S, et al.: Effects of irradiation of Er:YAG laser on quantity of endotoxin and microhardness of surface in exposed root after removal of calculus. Jpn J Conserv Dent. 41(6): 1009-1017, 1998 (in Japanese, English abstract).

44: Akiyama F, Aoki A, Miura-Uchiyama M, et al.: In vitro studies of the ablation mechanism of peridontopathic bacteria and decontamination effect on periodontally diseased root surfaces by erbium: yttrium-aluminium-garnet laser. Laser Med Sci. 26(2):193-204, 2011. Erratum in: Lasers Med Sci 26(2):277, 2011.

45: Sekine Y, Ebihara A, Takeda A, Suda H: Pulpal reaction in dog following cavity preparation by Er:YAG laser. Proc SPIE, 1984: 159-167, 1995.

46: Sonntag K, Klitzman B, Burkes E, et al.: Pulpal response to cavity preparation with the Er:YAG and Mark III free electron laser. Oral Surg Oral Med Oral Pathol Oral Radiol Endod. 81(6): 695702, 1996

47: Mizutani K, Aoki A, Takasaki A, et al.: Periodontal tissue healing following flap surgery using an Er:YAG laser in dogs. Lasers Surg Med. 38(4): 314324, 2006.

48: Schwartz F, Aoki A, Sculean A, et al.: In vivo effects of an Er:YAG laser, an ultrasonic system and scaling and root planning on the biocompatibility of periodontally diseased root surfaces in cultures of human PDL fibroblasts. Lasers Surg Med. 
33(2): 140-147, 2003.

49: Feist I, De Micheli G, Carneiro S, et al.: Adhesion and growth of cultured human gingival fibroblasts on periodontally involved root surfaces treated by Er:YAG laser. J Periodontol. 74(9): 1368-1375, 2003.

50: Belal M, Watanabe H, Ichinose S, Ishikawa I: Effect of Er:YAG laser combined with rh PDGF-BB on attachment of cultured fibroblasts to periodontally involved root surfaces. J Periodontol. 78(7): 13291341, 2007.

51: Maruyama H, Aoki A, Sasaki K, et al.: The effect of chemical and/or mechanical conditioning on the Er:YAG laser-treated root cementum: analysis of surface morphology and periodontal ligament fibroblast attachment. Lasers Surg Med. 40(3): 211 222, 2008

52: Schwarz F, Jepsen S, Herten M, et al.: Immunohistochemical characterization of periodontal wound healing following nonsurgical treatment with fluorescence controlled Er:YAG laser radiation in dogs. Lasers Surg Med. 39(5): 428-440, 2007.

53: Schwarz F, Sculean A, Georg T, Reich E: Periodontal treatment with an Er:YAG laser compared to scaling and root planning. A controlled clinical study. J Periodontol. 72(3): 361-367, 2001.

54: Schwarz F, Sculean A, Berakdar M, et al.: Peridontal treatment with an Er:YAG laser or scaling and root planing. A 2-year follow-up splitmouth study. J Periodontol. 74(5): 590-596, 2003.

55: Schwarz F, Sculean A, Berakdar M, et al.: Clinical evaluation of an Er:YAG laser combined with scaling and root planing for non-surgical periodontal treatment. A controlled prospective clinical study. J Clin Periodontol. 30(1): 26-34, 2003.

56: Sculean A, Schwarz F, Berakdar M, et al.:
Periodontal treatment with an Er:YAG laser compared to ultrasonic instrumentation: a pilot study. J Periodontol. 30(1): 966-973, 2004.

57: Crespi R, Cappare P, Toscanelli I, et al.: Effects of Er:YAG laser compared to ultrasonic scaler in periodontal treatment: a 2-year follow-up split-mouth clinical study. J Periodontol. 78(7): 1195-200, 2007.

58: Tomasi C, Schander K, Dahlen G, Wennstrom J: Short-term clinical and microbiologic effects of pocket debridement with an Er:YAG laser during periodontal maintenance. J Periodontol. 77(1): 111118, 2006.

59: Derdilopoulou F, Nonhoff J, Neumann K, Kielbassa A: Microbiologiccal findings after periodontal therapy using curettes, Er:YAG laser, sonic, and ultrasonic scalers. J Clin Periodontol. 34(7): 588-598, 2007.

60: Lopes B, Marcantonio R, Thompson G, et al.: Short-term clinical and immunologic effects of scaling and root planing with Er:YAG laser in chronic periodontitis. J Periodontol. 79(7):1158-1167, 2008.

61: Sculean A, Schwarz F, Berakdar M, et al.: Healing intrabony defects following surgical treatment with or without an Er:YAG laser. J Clin Periodontol. 31(8): 604-608, 2004.

62: Gaspric B, Skaleric U: Clinical evaluation of periodontal surgical treatment with an Er:YAG laser: 5year results. J Periodonotol. 78(10): 1864-1871, 2007.

63: The Research, Science and Therapy Committee of the American Academy of Periodontology: Lasers in periodontics (Academy report), authored by Cohen R and Ammons W, revised by Rossman J. J Periodontol. 73(10): 1231-1239, 2002.

64: Nagai S: Safety control in laser dentistry. J Jpn Soc Laser Dent. 19(3): 145-150, 2008 (in Japanese, English abstract).

These guidelines were translated from

Safety Guidelines for Laser Surgery and Medicine - 2011 version, supplement of The Journal of Japan Society for Laser Surgery and Medicine, Vol. 32, with the permission of the Safety Committee of the Japan Society for Laser Surgery and Medicine 